Elect. Comm. in Probab. 13 (2008), 45-53

\title{
A RELATION BETWEEN DIMENSION OF THE HARMONIC MEASURE, ENTROPY AND DRIFT FOR A RANDOM WALK ON A HYPERBOLIC SPACE
}

\author{
VINCENT LE PRINCE \\ IRMAR, Campus de Beaulieu, 35 042 Rennes cedex, France \\ email: vincent.leprince@univ-rennes1.fr
}

Submitted June 26, 2007, accepted in final form December 21, 2007

AMS 2000 Subject classification: 60G50, 20F67, 28D20, 28A78

Keywords: Random walk, hyperbolic space, harmonic measure, entropy, drift

\section{Abstract}

We establish in this paper an exact formula which links the dimension of the harmonic measure, the asymptotic entropy and the rate of escape for a random walk on a discrete subgroup of the isometry group of a Gromov hyperbolic space. This completes a result of [11], where only an upper bound for the dimension was proved.

\section{Introduction}

Let $\Gamma$ be a discrete subgroup of the isometry group of a Gromov hyperbolic space $X$ and $\mu$ a probability measure on $\Gamma$. The associated (right) random walk is the processus $\left(x_{n}\right)_{n \geq 0}$ defined by: $x_{0}=e$ and, for $n \geq 1, x_{n}=h_{1} \cdots h_{n}$, where $\left(h_{i}\right)_{i>0}$ is a sequence of independent random variables which take their values in $\Gamma$, with distribution $\mu$. Under some additional hypotheses, a point $o$ in $X$ being fixed, the trajectory $\left(x_{n} O\right)_{n \geq 0}$ converges almost surely to a point $x_{\infty}$ in the geometric boundary $\partial X$ of $X$. The distribution $\nu$ of $x_{\infty}$ is called the harmonic measure associated with the random walk.

The boundary $\partial X$ is equipped with a family of metrics $d_{a}$, where $a$ is a parameter. The aim of this paper is to established the following formula, which links the dimension of $\nu$ with respect to $d_{a}$, the asymptotic entropy $h(\mu)$ and the rate of escape $l(\mu)$ of the random walk (see Theorem 3.1 for a precise statement):

$$
\operatorname{dim} \nu=\frac{1}{\log a} \frac{h(\mu)}{l(\mu)} .
$$

This result completes the result obtained in [11], where only the bound from above in $(0.1)$ was proved. The main goal of the above mentioned paper was to show that the harmonic measure could be singular with respect to the Hausdorff measure; we refer to it for additional comments on the question of the dimension of the harmonic measure. 
The first result on this subject was obtained in [10] for a random walk on $S L(2, \mathbb{R})$ (which acts by isometries on the hyperbolic disc); a similar result was established in the case where $X$ is a tree in [8]. In our context of a hyperbolic space, and under the assumptions that $\mu$ is symmetric and finitely supported, the following result on the pointwise dimension is established in [2]:

$$
\lim _{r \rightarrow 0} \frac{\log \nu B_{a}(\xi, r)}{\log r}=\frac{1}{\log a} \frac{h(\mu)}{l(\mu)} \quad \nu-a . s .
$$

The dimension involved in our main theorem (3.1) is the box dimension and in this sense our result is weaker than (0.2). Indeed when $\log \nu B_{a}(\xi, r) / \log r$ converges a.s. to a constant, all reasonable notions of dimension coincide (see [13] or [12]). The interest of our result however lies in the fact that our hypotheses are the weakest possible - i.e. the weakest under which (0.1) makes sense: we just assume that $\mu$ admits a finite first moment.

The paper is organized as follows. In Section 1 we recall briefly the needed notions on random walks, hyperbolic spaces and dimensions of measures. We prove also in this first section a covering result on $\partial X$ which is necessary in order to compare the dimensions. In Section 2 we establish the lower bound for the dimension; which allows us to state our main result in Section 3 .

\section{Preliminaries and notations}

\subsection{Hyperbolic space}

The notion of Gromov hyperbolic space goes back to [7]. We refer to [4] and [6] for the definitions and properties that we state in this paragraph.

\section{Definition}

Let $(X, d)$ be a Gromov hyperbolic space. We denote by $B(x, R)$ the open ball with center $x$ and radius $R$ in $(X, d)$. Recall that the Gromov product (w.r.t. a point $w \in X$ ) on $X^{2}$ is defined by:

$$
(x \mid y)_{w}=\frac{1}{2}[d(w, x)+d(w, y)-d(x, y)] .
$$

The fact that $(X, d)$ is $\delta$-hyperbolic, for $\delta \geq 0$, is characterized by the fact that for every $w \in X$, for every $(x, y, z) \in X^{3}$,

$$
(x \mid z)_{w} \geq \min \left\{(x \mid y)_{w},(y \mid z)_{w}\right\}-\delta .
$$

We assume moreover that $(X, d)$ is proper and geodesic; we fix a base point $o$ in $X$ and denote by (.|.) the Gromov product with respect to $o$.

\section{Boundary}

The space $X$ is compactified by its geometric boundary $\partial X$. The action of the isometry group on $X$ extends to a continuous action on the boundary. We also denote by (.|.) the extension of the Gromov product to $\partial X$; on $X \cup \partial X$, (1.2) becomes:

$$
(x \mid z)_{w} \geq \min \left\{(x \mid y)_{w},(y \mid z)_{w}\right\}-2 \delta .
$$


Two distinct points of $X \cup \partial X$ can be joined by a geodesic. We shall also use the fact that for $x, y \in X \cup \partial X$, if $\left(x_{n}\right)_{n \geq 0}$ converges to $x$ and $\left(y_{n}\right)_{n \geq 0}$ converges to $y$, then

$$
(x \mid y)-2 \delta \leq \liminf _{n \rightarrow \infty}\left(x_{n} \mid y_{n}\right) \leq(x \mid y) .
$$

On this boundary a family of metrics $d_{a}, 1<a<a_{0}$, is defined, which have the following property: there exists a constant $\lambda>1$ such that $\forall x, y \in \partial X$

$$
\lambda^{-1} a^{-(x \mid y)} \leq d_{a}(x, y) \leq \lambda a^{-(x \mid y)} .
$$

We denote by $B_{a}(\xi, r)$ the open balls in $\left(\partial X, d_{a}\right)$; and, for $r>0, \log _{a} r=\frac{\log r}{\log a}$.

\section{Discrete subgroups}

Let $\Gamma$ be a group of isometries acting properly discontinuously on $X$. In particular, each orbit of $\Gamma$ in $X$ is discrete. Denote by $L_{\Gamma}$ the limit set of $\Gamma$. The group $\Gamma$ is said to be elementary if $L_{\Gamma}$ consists of at most two elements. If $G$ is non-elementary, $L_{\Gamma}$ is uncountable. The exponential growth rate of $\Gamma$ (in $X$ ) is:

$$
v(\Gamma)=\limsup _{R \rightarrow \infty} \frac{\log \operatorname{Card}\{g \in \Gamma \mid d(o, g o) \leq R\}}{R} .
$$

Another property we shall need is quasi-convex-cocompactness of $\Gamma$, which admits the following characterization ([3]): there exists $C>0$ such that every geodesic radius $\sigma$ joining $o$ to a point in $L_{\Gamma}$ stay at a distance lower than $C$ from $\Gamma o$.

Note that if $v(\Gamma)$ is finite and in addition $\Gamma$ is quasi-convex-cocompact, then the Hausdorff dimension (with respect to the metric $d_{a}$ ) of $L_{\Gamma}$ is equal to $v(\Gamma) / \log a$ (see [3]).

As important examples of quasi-convex-cocompact discrete groups, let us quote finitely generated hyperbolic groups acting on their Cayley graph (w.r.t. a finite set of generators) equipped with the word-length metric, or discrete convex cocompact groups of isometries acting on Riemaniann manifold with pinched sectional curvature.

\subsection{Random walk}

\section{Definition}

Given a countable group $\Gamma$ and a probability measure $\mu$ on $\Gamma$, the random walk associated to $\mu$ is defined as the homogeneous Markov processus on $\Gamma$ with initial state $e$ and transition probability $p(g, h)=\mu\left(g^{-1} h\right)$. Given a sequence $\left(h_{n}\right)_{n \geq 1}$ of independent random variables with law $\mu$, one defines a realization $\boldsymbol{x}=\left(x_{n}\right)_{n \geq 0}$ of this processus by $x_{0}=e$ and for $n>0$, $x_{n}=h_{1} \cdots h_{n}$. The sequence $\boldsymbol{x}=\left(x_{n}\right)_{n \geq 0}$ is called the trajectory of the random walk. We denote by $\mathbb{P}$ the law of this trajectory in $\Gamma^{\mathbb{N}}$. The distribution of the position $x_{n}$ of the trajectory at time $n$ is the convolution product $\mu^{n}=\mu * \cdots * \mu$, where $\mu$ appears $n$ times.

\section{Asymptotic entropy}

Recall that the entropy of a probability measure $m$ with countable support is defined by:

$$
H(m)=\sum_{g}-m(g) \log (m(g)) .
$$


The sequence $\left(H\left(\mu^{n}\right)\right)_{n}$ is subadditive so, assuming that the entropy of $\mu$ is finite, one can define the asymptotic entropy ([1]) of the random walk by

$$
h(\mu)=\lim _{n \rightarrow+\infty} \frac{1}{n} H\left(\mu^{n}\right) .
$$

Noting that

$$
H\left(\mu^{n}\right)=-\int \log \left(\mu^{n}\left(x_{n}\right)\right) d \mathbb{P},
$$

and using Kingman subadditive ergodic theorem, one gets ([5]) a $\mathbb{P}$-almost sure convergence:

$$
-\frac{1}{n} \log \left(\mu^{n}\left(x_{n}\right)\right) \longrightarrow h(\mu) \text {. }
$$

\section{Rate of escape}

Consider now a group $\Gamma$ acting by isometries on the metric space $(X, d)$. Assume that $\mu$ admits a finite first moment, that is to say

$$
\sum_{g \in \Gamma} \mu(g) d(o, g o)<\infty
$$

The rate of escape (also called drift) $l(\mu)$ of the random walk is defined, using once again a subadditivity property, by

$$
l(\mu)=\lim _{n \rightarrow+\infty} \frac{1}{n} d\left(o, x_{n} o\right) \quad \mathbb{P}-\text { a.s. } .
$$

Note that under the assumption that $v(\Gamma)<+\infty$, if $\mu$ admits a finite first moment, then it has also a finite entropy (see [9]), and so the asymptotic entropy of the random walk is well defined.

From now on, and unless otherwise specified, $\Gamma$ is a non-elementary group acting by isometries on a proper geodesic $\delta$-hyperbolic space $(X, d)$. We fix a metric $d_{a}$ on the boundary. We assume that $\Gamma$ acts properly discontinuously, is quasi-convex-cocompact, and that its exponential growth rate is finite. Moreover $\mu$ is a fixed probability measure on $\Gamma$, such that its support generates $\Gamma$ as a semigroup and which admits a finite first moment. We consider the random walk $\left(x_{n}\right)_{n \geq 0}$ associated with $\mu$; and we adopt the above notations.

\section{Asymptotic behaviour of the random walk}

Under the above hypotheses, the behaviour of the paths of the random walk is described by the following:

Theorem 1.1. ([9]) The trajectory $\left(x_{n} o\right)_{n \geq 0}$ converges $\mathbb{P}$-almost surely to an element $x_{\infty}$ in $\partial X$. More precisely, for $\mathbb{P}$-almost every trajectory $\left(x_{n} O\right)_{n \geq 0}$, there exists a geodesic radius $\sigma$ such that

$$
d\left(x_{n} o, \sigma(l(\mu) n)\right)=o(n)
$$


We denote by bnd the map (defined on a set of $\mathbb{P}$-measure 1 ) from $\Gamma^{\mathbb{N}}$ to $\partial X$ which associates $x_{\infty}$ to $x=\left(x_{n}\right)_{n \geq 0}$, and by $\nu$ the distribution of $x_{\infty}$, which we call the harmonic measure. In particular we have $\nu=\operatorname{bnd}(\mathbb{P})$.

Note that under these hypotheses, we have $l(\mu)>0$; as well as $h(\mu)>0$ (see [9]).

The support of $\nu$ is clearly included in the limit set $L_{\Gamma}$. It can be seen, using the minimality of $L_{\Gamma}$ and the fact that the measure $\nu$ is $\mu$-stationary, that the support of $\nu$ is in fact equal to $L_{\Gamma}$ (see e.g. [11]).

\subsection{Dimension of measures}

The Hausdorff dimension of a probability measure $m$ on $\partial X$ is defined by

$$
\operatorname{dim}_{H} m=\inf \left\{\operatorname{dim}_{H} A \mid m(A)=1\right\} .
$$

Note that this dimension is an invariant of the type of the measure $m$. Now let us define other notions of dimension of a measure we shall use and explore the relations between them (see [12] for an extensive presentation of this subject).

The first is the so-called box dimension. We denote by $N(A, r)$ the minimal number of balls of radius $r$ needed to cover a set $A \subset \partial X$. The lower and upper box dimensions of $A$ are defined respectively as

$$
\underline{\operatorname{dim}}_{B} A=\liminf _{r \rightarrow 0} \frac{\log N(A, r)}{\log 1 / r} \quad \text { and } \quad \overline{\operatorname{dim}}_{B} A=\limsup _{r \rightarrow 0} \frac{\log N(A, r)}{\log 1 / r},
$$

and the lower upper box dimensions of a probability measure $m$ as

$$
\begin{aligned}
& \underline{\operatorname{dim}}_{B} m=\lim _{\eta \rightarrow 0} \inf \left\{\underline{\operatorname{dim}}_{B} A \mid m(A) \geq 1-\eta\right\} \\
& \overline{\operatorname{dim}}_{B} m=\lim _{\eta \rightarrow 0} \inf \left\{\overline{\operatorname{dim}}_{B} A \mid m(A) \geq 1-\eta\right\} .
\end{aligned}
$$

When the upper and lower box dimensions coincide, we denote it by $\operatorname{dim}_{B} m$.

We focus now on the pointwise dimension. The lower and upper pointwise dimensions are defined for every $\xi$ in $X$ respectively by:

$$
\underline{\operatorname{dim}}_{P} m(\xi)=\liminf _{r \rightarrow 0} \frac{\log m B_{a}(\xi, r)}{\log r} \quad \text { and } \quad \overline{\operatorname{dim}}_{P} m(\xi)=\limsup _{r \rightarrow 0} \frac{\log m B_{a}(\xi, r)}{\log r}
$$

$\left(\operatorname{dim}_{P} m(\xi)\right.$ if both coincide).

In order to compare the box and pointwise dimensions, we shall need a covering property on the limit set. Recall that a cover of a set $A$ is said to have finite multiplicity $M$ if each point of $A$ lies in at most $M$ elements of the cover.

For a point $\xi \in \partial X$, we denote by $\sigma_{\xi}$ a (unit speed) geodesic ray joining $o$ to $\xi$. For the rest of this paragraph, we omit to precise that the properties are satisfied for $r$ small enough. The following lemma establishes a link between shadows and balls on $\partial X$.

Lemma 1.2. For $\xi, \xi^{\prime}$ in $\partial X$; for every $R_{1} \in \mathbb{R}, R_{2}>0$, if

$$
d\left(\sigma_{\xi}\left(-\log _{a} r+R_{1}\right), \sigma_{\xi^{\prime}}\left(-\log _{a} r+R_{1}\right)\right)<R_{2}
$$


then we have

$$
d_{a}\left(\xi, \xi^{\prime}\right)<r \times \lambda a^{-R_{1}+R_{2} / 2+4 \delta} .
$$

Conversely assume that $d_{a}\left(\xi, \xi^{\prime}\right)<r$; then, for every $R_{1} \in \mathbb{R}$,

$$
d\left(\sigma_{\xi}\left(-\log _{a} r+R_{1}\right), \sigma_{\xi_{i}}\left(-\log _{a} r+R_{1}\right)\right)<2 \log _{a} \lambda+4\left|R_{1}\right|+8 \delta .
$$

Proof. If $d\left(\sigma_{\xi}\left(-\log _{a} r+R_{1}\right), \sigma_{\xi^{\prime}}\left(-\log _{a} r+R_{1}\right)\right)<R_{2}$, then by (1.1),

$$
\left(\sigma_{\xi}\left(-\log _{a} r+R_{1}\right) \mid \sigma_{\xi^{\prime}}\left(-\log _{a} r+R_{1}\right)\right)>-\log _{a} r+R_{1}-R_{2} / 2 .
$$

Besides, by (1.4), we have

$$
\left(\xi \mid \sigma_{\xi}\left(-\log _{a} r+R_{1}\right)\right) \geq \liminf _{n \rightarrow+\infty}\left(\sigma_{\xi}(n) \mid \sigma_{\xi}\left(-\log _{a} r+R_{1}\right)\right)=-\log _{a} r+R_{1},
$$

as well as $\left(\sigma_{\xi^{\prime}}\left(-\log _{a} r+R_{1}\right) \mid \xi^{\prime}\right) \geq-\log _{a} r+R_{1}$. Using (1.3), this implies

$$
\left(\xi \mid \xi^{\prime}\right)>-\log _{a} r+R_{1}-R_{2} / 2-4 \delta,
$$

and the result follows from $(1.5)$.

Conversely, if $d_{a}\left(\xi, \xi^{\prime}\right)<r$, we have $\lambda^{-1} a^{-\left(\xi \mid \xi^{\prime}\right)}<r$, and so $\left(\xi \mid \xi^{\prime}\right)>-\log _{a} r-\log _{a} \lambda$. Using (1.3), this implies, for $R_{1} \in \mathbb{R}$,

$$
\left(\sigma_{\xi}\left(-\log _{a} r+R_{1}\right) \mid \sigma_{\xi^{\prime}}\left(-\log _{a} r+R_{1}\right)\right)>-\log _{a} r-\log _{a} \lambda-\left|R_{1}\right|-4 \delta,
$$

and so

$$
\begin{aligned}
d\left(\sigma_{\xi}\left(-\log _{a} r+R_{1}\right), \sigma_{\xi^{\prime}}\left(-\log _{a} r+R_{1}\right)\right)< & 2\left(-\log _{a} r+R_{1}\right) \\
& -2\left(-\log _{a} r-\log _{a} \lambda-\left|R_{1}\right|-4 \delta\right)
\end{aligned}
$$

which gives the result.

Proposition 1.3. There exists a constant $M>0$ such that every subset $A$ of $L_{\Gamma}$ admits, for every $r>0$, a covering by balls centered in $A$, of radius $r$, with multiplicity $M$.

Proof. Let $A$ be a subset of $L_{\Gamma}$ and $r>0$. We set

$$
T=-\log _{a} r+\log _{a} \lambda+C+4 \delta,
$$

where the constant $C$ comes from the caracterization of quasi-convex-cocompactness (see par. 1.1). Since $\Gamma$ is quasi-convex-cocompact, for each $\xi \in A$, there exists $g_{r, \xi} \in \Gamma$ such that

$$
d\left(\sigma_{\xi}(T), g_{r, \xi} O\right) \leq C .
$$

Denote by $\left\{g_{i}, i \in I\right\}$ the set of $g_{r, \xi}, \xi \in A$, and for each $i \in I$ choose $\xi_{i} \in A$ such that $g_{r, \xi_{i}}=g_{i}$.

Now, for a $\xi \in A$, take $i \in I$ such that $g_{r, \xi}=g_{i}$. We have

$$
d\left(\sigma_{\xi_{i}}(T), \sigma_{\xi}(T)\right) \leq 2 C
$$

So by the use Lemma 1.2 ,

$$
d_{a}\left(\xi, \xi_{i}\right)<r \times \lambda a^{-\left(\log _{a} \lambda+C+4 \delta\right)+2 C / 2+4 \delta}=r,
$$


and $\xi \in B_{a}\left(\xi_{i}, r\right)$. Therefore $\left\{B_{a}\left(\xi_{i}, r\right), i \in I\right\}$, is a covering of $A$.

Now let us prove that this covering has finite multiplicity (not depending on $r$ ).

For $\xi \in \partial X$, let be $i \in I$ such that $\xi \in B_{a}\left(\xi_{i}, r\right)$. We have, by the second part of Lemma 1.2 ,

$$
d\left(\sigma_{\xi}(T), \sigma_{\xi_{i}}(T)\right)<2 \log _{a} \lambda+4\left|\log _{a} \lambda+C+4 \delta\right|+8 \delta,
$$

and so

$$
d\left(\sigma_{\xi}(T), g_{i}\right) \leq d\left(\sigma_{\xi}(T), \sigma_{\xi_{i}}(T)\right)+d\left(\sigma_{\xi_{i}}(T), g_{i}\right)<D
$$

where $D$ is a constant (i.e. does not depend on $r$ but only on $C, \delta$ and $a$ ). But the number of elements in $\Gamma$ that are at a distance lower than $D$ from $\sigma_{\xi}(T)$ is bounded by a constant $M$, so that the covering just constructed has finite multiplicity $M$.

This covering property of the boundary has the following consequence (see [12]):

Proposition 1.4. Assume that for a probability measure $m$ on $L_{\Gamma}$, there exists $d$ such that $\overline{\operatorname{dim}}_{P} m(\xi) \leq d$ for $m$-almost every $\xi$. Then $\overline{\operatorname{dim}}_{B} m \leq d$.

We have also (see [12]) $\operatorname{dim}_{H} m \leq \underline{\operatorname{dim}}_{B} m$.

\section{Lower bound for the dimension}

Proposition 2.1. The harmonic measure $\nu$ associated to the random walk satisfies

$$
\underline{\operatorname{dim}}_{B} \nu \geq \frac{1}{\log a} \frac{h(\mu)}{l(\mu)} .
$$

In this section, in order to simplify the notations, we write $d$ for the dimension $\underline{\operatorname{dim}}_{B} \nu$ and $l$ and $h$ for respectively $l(\mu)$ and $h(\mu)$. We first need the following lemma, which is a direct consequence of the definition of this dimension.

Lemma 2.2. For every $\eta>0$, there exists a sequence $\left(r_{k}\right)_{k>0}$ which converges to zero and such that for every $k>0$, there exists a set $E_{\eta, k} \subset \partial X$ which satisfies:

$$
\log \operatorname{Card} E_{\eta, k} \leq(d+1 / k) \log \left(1 / r_{k}\right)
$$

and

$$
\nu\left(\bigcup_{\xi \in E_{\eta, k}} B_{a}\left(\xi, r_{k}\right)\right) \geq 1-\eta .
$$

Now let us prove Proposition 2.1. Fix $\epsilon>0, \eta>0$ and set

$$
\Omega_{\epsilon}^{n}=\left\{\boldsymbol{x} \mid \mu^{n}\left(x_{n}\right) \leq e^{-n(h-\epsilon)}, d\left(\sigma_{b n d(\boldsymbol{x})}(n l), x_{n} O\right) \leq n \epsilon\right\},
$$

where $\sigma_{b n d(\boldsymbol{x})}$ denotes a geodesic joining $o$ to $x_{\infty}=b n d(\boldsymbol{x})$. In view of the behaviour of the random walk (Theorem 1.1 and (1.7)), there exists $N_{\epsilon, \eta}$, such that for every $n \geq N_{\epsilon, \eta}$, $\mathbb{P}\left(\Omega_{\epsilon}^{n}\right) \geq 1-\eta$. Then we take $\left(r_{k}\right)_{k>0}$ as in Lemma 2.2 , set $n_{k}=\left[\left(-\log _{a} r_{k}\right) / l\right]$ (integer part) and, with the notations of this lemma,

$$
\Pi_{\epsilon, \eta}^{k}=\Omega_{\epsilon}^{n_{k}} \bigcap b n d^{-1}\left(\bigcup_{\xi \in E_{\eta, k}} B_{a}\left(\xi, r_{k}\right)\right) \text {. }
$$


We immediately get, for $k$ such that $n_{k} \geq N_{\epsilon, \eta}, \mathbb{P}\left(\Pi_{\epsilon, \eta}^{k}\right) \geq 1-2 \eta$, and

$$
\mathbb{P}\left(\Pi_{\epsilon, \eta}^{k}\right) \leq \sum_{\xi \in E_{\eta, k}} \mathbb{P}\left(\Omega_{\epsilon}^{n_{k}} \cap b n d^{-1}\left(B_{a}\left(\xi, r_{k}\right)\right)\right) .
$$

Let us fix $\xi \in E_{\eta, k}$ and assume that $\boldsymbol{x} \in \Omega_{\epsilon}^{n_{k}} \cap b n d^{-1}\left(B_{a}\left(\xi, r_{k}\right)\right)$. We have $d(\operatorname{bnd}(\boldsymbol{x}), \xi)<r_{k}$, so that, by Lemma 1.2 ,

$$
d\left(\sigma_{b n d(\boldsymbol{x})}\left(-\log _{a} r_{k}\right), \sigma_{\xi}\left(-\log _{a} r_{k}\right)\right) \leq 2 \log _{a} \lambda+8 \delta .
$$

Besides

$$
d\left(\sigma_{b n d(\boldsymbol{x})}\left(n_{k} l\right), x_{n_{k}} o\right) \leq n_{k} \epsilon
$$

and, since $\left|n_{k} l-\left(-\log _{a} r_{k}\right)\right| \leq l$, this yields

$$
d\left(\sigma_{\xi}\left(n_{k} l\right), x_{n_{k}} o\right) \leq n_{k} \epsilon+2 l+2 \log _{a} \lambda+8 \delta,
$$

and $d\left(\sigma_{\xi}\left(n_{k} l\right), x_{n_{k}} o\right) \leq 2 n_{k} \epsilon$ for $k$ big enough.

So we get, for a $\xi \in E_{\eta, k}$,

$$
\mathbb{P}\left(\Omega_{\epsilon}^{n_{k}} \cap b n d^{-1}\left(B_{a}\left(\xi, r_{k}\right)\right)\right) \leq \mathbb{P}\left\{\boldsymbol{x} \mid x_{n_{k}} \in B\left(\sigma_{\xi}\left(n_{k} l\right), 2 n_{k} \epsilon\right), \mu^{n_{k}}\left(x_{n_{k}}\right) \leq e^{-n_{k}(h-\epsilon)}\right\},
$$

and since the distribution of $x_{n_{k}}$ under $\mathbb{P}$ is $\mu^{n_{k}}$, this implies

$$
\begin{aligned}
\mathbb{P}\left(\Omega_{\epsilon}^{n_{k}} \cap b n d^{-1}\left(B_{a}\left(\xi, r_{k}\right)\right)\right) & \leq \mu^{n_{k}}\left\{g \in \Gamma \mid g \in B\left(\sigma_{\xi}\left(n_{k} l\right), 2 n_{k} \epsilon\right), \mu^{n_{k}}(g) \leq e^{-n_{k}(h-\epsilon)}\right\} \\
& \leq \operatorname{Card} B\left(\sigma_{\xi}\left(n_{k} l\right), 2 n_{k} \epsilon\right) \times e^{-n_{k}(h-\epsilon)} .
\end{aligned}
$$

Now we use the fact that, since $v(\Gamma)<+\infty$, there exists a constant $V>0$ such that for every $x \in X$ and every $R>0$,

$$
\text { Card }\{g \in \Gamma \mid d(x, g o) \leq R\} \leq V^{R} .
$$

So the above inequality, (2.1) and the fact that $\operatorname{Card} E_{\eta, k} \leq\left(1 / r_{k}\right)^{d+1 / k}$ give

$$
1-2 \eta \leq \mathbb{P}\left(\Pi_{\epsilon, \eta}^{k}\right) \leq\left(\frac{1}{r_{k}}\right)^{d+1 / k} V^{2 n_{k} \epsilon} e^{-n_{k}(h-\epsilon)},
$$

therefore

$$
\frac{\log (1-2 \eta)}{n_{k}} \leq \frac{\left(\log _{a} r_{k}\right) / l}{n_{k}} l(d+1 / k)+\frac{2 n_{k} \epsilon}{n_{k}} \log V-(h-\epsilon) .
$$

Finaly we note that $\lim _{k \rightarrow \infty} \frac{\left(\log _{a} r_{k}\right) / l}{n_{k}}=1$; so, doing $k \rightarrow \infty$, and since $\epsilon$ and $\eta$ are arbitrary, this achieves the proof.

\section{Conclusion}

It was shown in [11] that in our context, the harmonic measure satisfies:

$$
\overline{\operatorname{dim}}_{P} \nu(\xi) \leq \frac{1}{\log a} \frac{h(\mu)}{l(\mu)} \quad \nu-a . s .
$$

So, taking into account the result of Section 2 and Proposition 1.4 , we get the following 
Theorem 3.1. Under our hypotheses, the harmonic measure satisfies:

$$
\operatorname{dim}_{B} \nu=\frac{1}{\log a} \frac{h(\mu)}{l(\mu)} .
$$

Remark 3.2. Under our hypotheses, it is known that $h(\mu)>0$ (see [9]); so as a consequence of Theorem 3.1 we have $\operatorname{dim}_{B} \nu>0$.

\section{References}

[1] A. Avez, Entropie des groupes de type fini, C. R. Acad. Sci. Paris, Sér. A, vol. 275 (1972), p $1363-1366$.

[2] S. Blachère, P. Haïssinsky, and P. Mathieu, Harmonic measures versus quasiconformal measures for hyperbolic groups, preprint (2007).

[3] M. Coornaert, Mesures de Patterson-Sullivan sur le bord d'un espace hyperbolique au sens de Gromov, Pacific Journal of Mathematics, vol.159 (1993), p 241-270. MR1214072

[4] M. Coornaert, T. Delzant, A. Papadopoulos, Géométrie et théorie des groupes : les groupes hyperboliques de Gromov, Lecture Notes in Math. 1441, Springer, 1990. MR1075994

[5] Y. Derriennic, Quelques applications du théorème ergodique sous-additif, Asterisque 74 (1980), p 183-201. MR0588163

[6] E. Ghys, P. De La Harpe (eds.), Sur les Groupes Hyperboliques d'après Mikhael Gromov, Birkhäuser, Basel, 1990. MR1086648

[7] M. Gromov, Hyperbolic groups, Essays in Group Theory (S.M. Gersten, ed.), MSRI Publ., vol. 8, Springer, New York, 1987, p 75-263. MR0919829

[8] V. A. Kaimanovich, Hausdorff dimension of the harmonic measure on trees, Ergod. Th. \& Dynam. Sys. (1998), p 631-660. MR1631732

[9] V. A. Kaimanovich, The Poisson formula for groups with hyperbolic properties, Annals of Mathematics, vol. 152 (2000), p 659-692. MR1815698

[10] F. Ledrappier, Une relation entre entropie, dimension et exposant pour certaines marches aléatoires, C. R. Acad. Sci. Paris Sér. I Math., vol. 296 (1983), p. 369-372. MR0699165

[11] V. Le Prince, Dimensional properties of the harmonic measure for a random walk on a hyperbolic group, Trans. of the AMS 359 (2007), p 2881-2898. MR2286061

[12] Ya. B. Pesin, Dimension theory in dynamical systems, Chicago Lect. Notes in Math. (1997). MR1489237

[13] L. S. Young, Dimension, entropy and Lyapunov exponents, Ergod. Th. \& Dynam. Sys. (1982), p 109-124. MR0684248 